\title{
Stability of Non-Hyperbolic Equilibrium Point for Polynomial System of Differential Equations
}

\author{
Vahidin Hadžiabdić, Midhat Mehuljić, Jasmin Bektešević \\ Faculty of Mechanical Engineering, Department of Mathematics / University of Sarajevo, \\ Sarajevo, Bosnia and Herzegovina
}

\begin{abstract}
In this paper, a polynomial system of plane differential equations is observed. The stability of the non-hyperbolic equilibrium point was analyzed using normal forms. The nonlinear part of the differential equation system is simplified to the maximum. Two nonlinear transformations were used to simplify first the quadratic and then the cubic part of the system of equations.
\end{abstract}

Keywords - Equilibrium Point, Stability, Nullcline, Normal forms.

\section{Introduction}

Consider a system of differential equations of form

$$
\left\{\begin{array}{l}
\dot{x}=a x+b y+f(x, y), \\
\dot{y}=c x+d y+g(x, y),
\end{array}\right.
$$

where $f(x, y)$ and $g(x, y)$ are polynomials of degree greater than one. In the case of hyperbolic points of equilibrium of the nonlinear system of differential equations, the Hartman-Grobman theorem solves the stability problem around the point of equilibrium. When the equilibrium point is non-hyperbolic, then the stability testing around that point, based on the

DOI: 10.18421/TEM103-52

https://doi.org/10.18421/TEM103-52

Corresponding author: Vahidin Hadžiabdić,

Faculty of Mechanical Engineering, Department of Mathematics / University of Sarajevo, Sarajevo, Bosnia and Herzegovina.

Email: hadziabdic@mef.unsa.ba

Received: 23 April 2021.

Revised: 01 July 2021.

Accepted: 09 July 2021.

Published: 27 August 2021.

(c))BY-Nc-ND 2021 Vahidin Hadžiabdić, Midhat Mehuljić \& Jasmin Bektešević; published by UIKTEN. This work is licensed under the Creative Commons AttributionNonCommercial-NoDerivs 4.0 License.

The article is published with Open Access at www.temjournal.com
Center Manifold Theorem, is reduced to a stability test on the center manifold, which is in general a dimension smaller than or equal to the dimension of the initial system.

The following system will be considered in this paper:

$\left\{\begin{array}{l}\dot{x}=y+x^{2}-x^{3}+x y^{2}-y^{3} \\ \dot{y}=x^{2}-2 x y+x^{3}-x^{2} y\end{array}\right.$,

A very powerful tool in examining nonlinear systems where non-hyperbolic points of equilibrium occur are normal forms. For a generalization of the general results from Sternberg and results from Hartman, see [1].

A fairly effective, but often hardly practical, way to test the stability of non-hyperbolic points is by using Lyapun's function. If Lyapun's function is found then it is elegant proof. Finding of invariant closed surface and stability of non-hyperbolic point for a particular class of polynomial systems was observed in [2].

Using the Normal Form Theory and Center Manifold Theorem in [3], Hopf bifurcation, as well as the stability of the periodic solution, were investigated for the predator-prey model.

If a system of form (1) having a non-hyperbolic equilibrium point $O(0,0)$, it can be written in the form

$\left\{\begin{array}{c}\dot{x}=y \\ \dot{y}=\alpha_{k} x^{k}(1+h(x))+\beta_{n} x^{n} y(1+g(x))+y^{2} R(x, y),\end{array}\right.$

Where $h(x), g(x)$ and $R(x, y)$ are analytic in a neighborhood of the origin, $h(0)=g(0)=0, k \geq 2$, $\alpha_{k} \neq 0$, and $n \geq 1$, see [5]. Also in [5] see the following theorem.

Theorem 1. Let $k=2 m$ with $m \geq 1$ in the above system. Then the origin is

a) a cusp if $\beta_{n}=0$ and also if $\beta_{n} \neq 0$ and $n \geq m$,

b) a saddle-node if $\beta_{n} \neq 0$ and $n<m$.

It should be noted that the above theorem also applies to the more general systems with respect to this system, which we observe. Namely, it is not necessary for the system to be polynomial. 


\section{Stability of Equilibrium Points}

The equilibrium points of system (2) are obtained by solving the algebraic system of equations:

$\left\{\begin{array}{l}y+x^{2}-x^{3}+x y^{2}-y^{3}=0 \\ x^{2}-2 x y+x^{3}-x^{2} y=0\end{array}\right.$

From the second equation of system (3) we have that $x=0$. Putting it into the first equation we get three points of equilibrium: $O(0,0), A(0,-1), B(0,1)$.

Also, if we express y from second equation, we have that

$$
y=\frac{x^{2}+x}{x+2}
$$

and after fitting into the first equation of (3) and simplifying we get that

$$
-x\left(x^{5}+4 x^{4}-3 x^{3}-10 x^{2}+16 x-4\right)=0 .
$$

From here we see that the point $O(0,0)$ is a double solution of system (3). Based on Descartes' signchange rule, we conclude that the last equation has one positive zero. In addition, based on the same rule, we conclude that the last equation has 0,2 , or 4 negative zeros. Purely algebraically, it is difficult to calculate the exact values of negative real roots.

Using the Math package, we get that there are three more real equilibrium points whose values are approximately equal : $C(1.67,1.22), D(-1.50,1.53)$, $E(-0.31,-0.12)$. Figure 1 shows the vector field of the system (3).

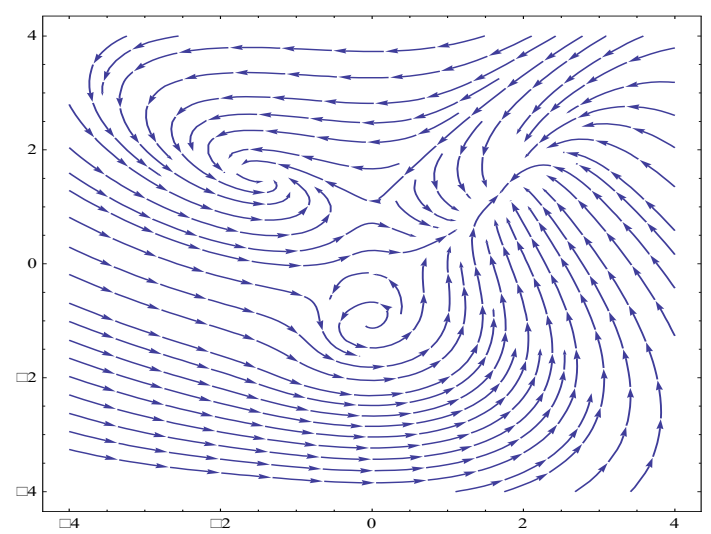

Figure 1. Vector field for system (2)

The curves given on the left side of system (3) represent nullclines. Figure 2 shows the nullclines as well as all 6 equilibrium points of the system (3). The curve colored in red represents $x$-nullclines, and the curve colored in blue represents $y$-nullclines. Nuclines themselves do not provide a solution to the global dynamics of the system, but they can help us to anticipate its long-term behavior, see [4].

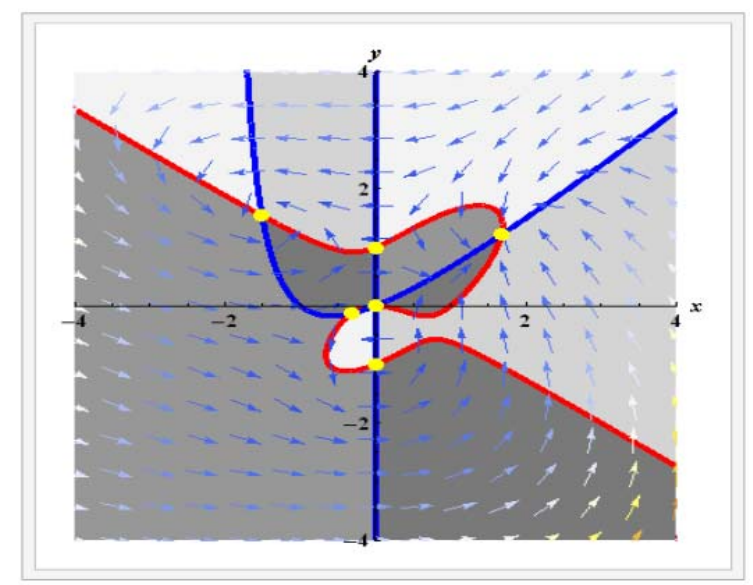

Figure 2. The nullclines of system (3)

Jacob's matrix of system (3) is given by

$$
D_{f}=\left(\begin{array}{cc}
2 x-3 x^{2}+y^{2} & 1+2 x y-3 y^{2} \\
2 x-2 y+3 x^{2}-2 x y & -2 x-x^{2}
\end{array}\right)
$$

Lemma 1: The equilibrium points $A(0,-1)$ and $B(0,1)$ are unstable. The point $A(0,-1)$ is the source, and the point $B(0,1)$ is the saddle.

Proof: We just get that it is

$$
D_{f}(A)=\left(\begin{array}{cc}
1 & -2 \\
2 & 0
\end{array}\right) \text {, }
$$

and the eigenvalues of this matrix are given by:

$$
\lambda_{1,2}=\frac{1}{2} \pm \frac{\sqrt{15}}{2} i
$$

We see that both eigenvalues have a positive real part, so point $A(0,-1)$ is the source.

It's similar

$$
D_{f}(B)=\left(\begin{array}{cc}
1 & -2 \\
-2 & 0
\end{array}\right)
$$

which has eigenvalues

$$
\lambda_{1,2}=\frac{1}{2} \pm \frac{\sqrt{17}}{2}
$$

The eigenvalues are of different sign, so the point $B(0,1)$ is the saddle.

TheFigure 3 shows the trajectories of the system (3) using the Math software package.

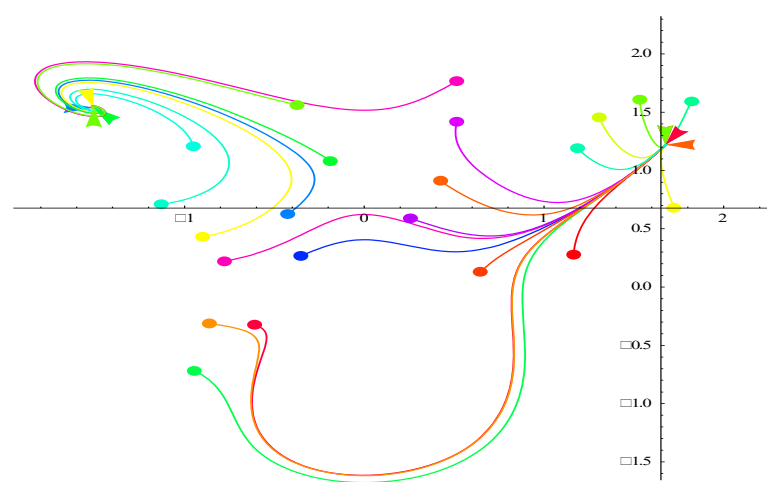

Figure 3. Trajectories of system (3) 
Since,

$$
D_{f}(O)=\left(\begin{array}{ll}
0 & 1 \\
0 & 0
\end{array}\right)
$$

$\left[\begin{array}{c}y_{1}^{2} \\ y_{1}^{2}-2 y_{1} y_{2}\end{array}\right]=$

It is not difficult to see that both eigenvalues of $O(0,0)$ are equal to 0 . So, this is a non-hyperbolic fixed point. Below, we use normal form theory and Center Manifold Theorem to examine the local stability of point $O(0,0)$.

System (2), which is in vector form

$$
\dot{x}=A x+f_{2}(x)+f_{3}(x)+0\left(|x|^{4}\right)
$$

we want to transforme, using normal forms, so that the first equation of this system is of the form

$\dot{x}=y+0\left(|x|^{4}\right)$.

We have that

$A=\left[\begin{array}{ll}0 & 1 \\ 0 & 0\end{array}\right], \quad \boldsymbol{f}_{2}(\boldsymbol{x})=\left[\begin{array}{c}x^{2} \\ x^{2}-2 x y\end{array}\right]$

and $\boldsymbol{f}_{\mathbf{3}}(\boldsymbol{x})=\left[\begin{array}{c}-x^{3}+x y^{2}-y^{3} \\ 3 x^{3}+x^{2} y\end{array}\right]$.

Using a shift

$x=y+h_{2}(y)$,

where $\boldsymbol{h}_{\mathbf{2}}(\boldsymbol{y})=0\left(|\boldsymbol{y}|^{2}\right)$, as $|\boldsymbol{y}| T 0$,

we will eliminate the quadratic terms from the first equation of system (2). Based on (6), we have that system

$\dot{\boldsymbol{x}}=A \boldsymbol{x}+\boldsymbol{f}_{2}(\boldsymbol{x})$

where

$$
\boldsymbol{f}_{2}(\boldsymbol{x})=\left[\begin{array}{c}
x^{2} \\
x^{2}-2 x y
\end{array}\right]
$$

can write in the form

$\dot{\boldsymbol{y}}=A \boldsymbol{y}+\boldsymbol{g}_{\mathbf{2}}(\boldsymbol{y})+0\left(|\boldsymbol{y}|^{3}\right)$, as $|\boldsymbol{y}| T 0$, where

$\boldsymbol{g}_{2}(\boldsymbol{y})=A \widetilde{\boldsymbol{h}_{2}}(\boldsymbol{y})-D \widetilde{\boldsymbol{h}_{2}}(\boldsymbol{y}) A+\boldsymbol{f}_{2}(\boldsymbol{y})$,

and

$\boldsymbol{h}_{\mathbf{2}}(\boldsymbol{y})=\widetilde{\boldsymbol{h}_{\mathbf{2}}}(\boldsymbol{y})+0\left(|\boldsymbol{y}|^{3}\right)$, as $|\boldsymbol{y}| 70$.

In the next step, we want to determine $\boldsymbol{h}_{\mathbf{2}}(\boldsymbol{y})$ so we get $\boldsymbol{g}_{\mathbf{2}}(\boldsymbol{y})$ as easy as possible. If we insert

$$
\widetilde{\boldsymbol{h}_{2}}(\boldsymbol{y})=\left[\begin{array}{c}
\alpha_{20} y_{1}^{2}+\alpha_{11} y_{1} y_{2}+\alpha_{02} y_{2}^{2} \\
\beta_{20} y_{1}^{2}+\beta y_{1} y_{2}+\beta_{02} y_{2}^{2}
\end{array}\right]
$$

into (9), and then (9) into (8), then

$$
\begin{aligned}
& \boldsymbol{g}_{2}(\boldsymbol{y})=\left[\begin{array}{ll}
0 & 1 \\
0 & 0
\end{array}\right]\left[\begin{array}{l}
\alpha_{20} y_{1}^{2}+\alpha_{11} y_{1} y_{2}+\alpha_{02} y_{2}^{2} \\
\beta_{20} y_{1}^{2}+\beta_{11} y_{1} y_{2}+\beta_{02} y_{2}^{2}
\end{array}\right]- \\
& {\left[\begin{array}{ll}
2 \alpha_{20} y_{1}+a_{11} y_{2} & \alpha_{11} y_{1}+2 \alpha_{02} y_{2} \\
2 \beta_{20} y_{1}+\beta_{11} y_{2} & \beta_{11} y_{1}+2 \beta_{02} y_{2}
\end{array}\right]\left[\begin{array}{ll}
0 & 1 \\
0 & 0
\end{array}\right]\left[\begin{array}{l}
y_{1} \\
y_{2}
\end{array}\right]+}
\end{aligned}
$$

$\left[\begin{array}{c}\left(\beta_{20}+1\right) y_{1}^{2}+\left(\beta_{11}+2 \alpha_{20}\right) y_{1} y_{2}+\left(\beta_{02}+\alpha_{11}\right) y_{2}^{2} \\ y_{1}^{2}-\left(2 \beta_{20}+2\right) y_{1} y_{2}+\beta_{11} y_{2}^{2}\end{array}\right]$.

If we choose the coefficients so that

$\beta_{20}=-1, \beta_{11}=-2 \alpha_{20}, \beta_{02}=-\alpha_{11}, \beta_{11}=0$, then

$\boldsymbol{g}_{2}(\boldsymbol{y})=\left[\begin{array}{c}0 \\ y_{1}^{2}\end{array}\right]$.

So relation (7) now becomes

$\dot{\boldsymbol{x}}=A \boldsymbol{x}+\left[\begin{array}{c}0 \\ x^{2}\end{array}\right]+0\left(|\boldsymbol{x}|^{3}\right)$.

In this way, we eliminated the square terms from the first equation of system (2). Using a similar technique, we will now eliminate cubic members from the first equation of system (2).

Using the shift now

$x=y+h_{3}(y)$,

We have that

$\dot{\boldsymbol{x}}=\dot{\boldsymbol{y}}+D \boldsymbol{h}_{\mathbf{3}}(\boldsymbol{y}) \dot{\boldsymbol{y}}$.

Using (13) we have

$$
\left[I+D \boldsymbol{h}_{3}(\boldsymbol{y})\right] \dot{\boldsymbol{y}}=A \boldsymbol{y}+A \boldsymbol{h}_{3}(\boldsymbol{y})+\boldsymbol{f}_{\mathbf{3}}(\boldsymbol{y}) .
$$

From there it is

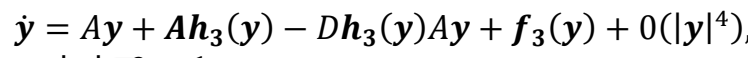

as $|\boldsymbol{y}| 70$, where

$\boldsymbol{h}_{\mathbf{3}}(\boldsymbol{y})=\widetilde{\boldsymbol{h}_{\mathbf{3}}}(\boldsymbol{y})+0\left(|\boldsymbol{y}|^{4}\right)$, as $|\boldsymbol{y}| 70$.

We know it is

$\boldsymbol{f}_{\mathbf{3}}(\boldsymbol{y})=\left[\begin{array}{c}-y_{1}^{3}+y_{1} y_{2}^{2}-y_{2}^{3} \\ 3 y_{1}^{3}+y_{1}^{2} y_{2}\end{array}\right]$. Relation (14) can be

written in the form

$\dot{\boldsymbol{y}}=A \boldsymbol{y}+\boldsymbol{g}_{\mathbf{3}}(\boldsymbol{y})+0\left(|\boldsymbol{y}|^{4}\right)$, as $|\boldsymbol{y}| 70$,

Where

$$
\begin{aligned}
& g_{3}(y)=A \widetilde{h_{3}}(y)-D \widetilde{h_{3}}(y) A y+f_{3}(y) \text {, and } \\
& \boldsymbol{h}_{\mathbf{3}}(\boldsymbol{y})=\widetilde{\boldsymbol{h}_{\mathbf{3}}}(\boldsymbol{y})+0\left(|\boldsymbol{y}|^{4}\right) \text {, as }|\boldsymbol{y}| T 0 \text {. }
\end{aligned}
$$

Since

$\boldsymbol{h}_{\mathbf{3}}(\boldsymbol{y})=\left[\begin{array}{l}\alpha_{30} y_{1}^{3}+\alpha_{21} y_{1}^{2} y_{2}+\alpha_{12} y_{1} y_{2}^{2}+\alpha_{03} y_{2}^{3} \\ \beta_{30} y_{1}^{3}+\beta_{21} y_{1}^{2} y_{2}+\beta_{12} y_{1} y_{2}^{2}+\beta_{03} y_{2}^{3}\end{array}\right]$,

then

$$
\begin{gathered}
\boldsymbol{g}_{3}(\boldsymbol{y})=\left[\begin{array}{ll}
0 & 1 \\
0 & 0
\end{array}\right]\left[\begin{array}{l}
\alpha_{30} y_{1}^{3}+\alpha_{21} y_{1}^{2} y_{2}+\alpha_{12} y_{1} y_{2}^{2}+\alpha_{03} y_{2}^{3} \\
\beta_{30} y_{1}^{3}+\beta_{21} y_{1}^{2} y_{2}+\beta_{12} y_{1} y_{2}^{2}+\beta_{03} y_{2}^{3}
\end{array}\right] \\
- \\
{\left[\begin{array}{cc}
3 \alpha_{30} y_{1}^{2}+2 \alpha_{21} y_{1} y_{2}+\alpha_{12} y_{2}^{2} & \alpha_{21} y_{1}^{2}+2 \alpha_{12} y_{1} y_{2}+3 \alpha_{03} y_{2}^{2} \\
3 \beta_{30} y_{1}^{2}+2 \beta_{21} y_{1} y_{2}+\beta y_{2}^{2} & \beta_{21} y_{1}^{2}+2 \beta_{12} y_{1} y_{2}+3 \beta_{03} y_{2}^{2}
\end{array}\right]}
\end{gathered}
$$


$\left[\begin{array}{ll}0 & 1 \\ 0 & 0\end{array}\right]\left[\begin{array}{l}y_{1} \\ y_{2}\end{array}\right]+\left[\begin{array}{c}-y_{1}^{3}+y_{1} y_{2}^{2}-y_{2}^{3} \\ 3 y_{1}^{3}+y_{1}^{2} y_{2}\end{array}\right]=$

$\left[\begin{array}{c}\beta_{30} y_{1}^{3}+\beta_{21} y_{1}^{2} y_{2}+\beta_{12} y_{1} y_{2}^{2}+\beta_{03} y_{2}^{3} \\ 0\end{array}\right]$

$\left[\begin{array}{c}3 \alpha_{30} y_{1}^{2} y_{2}+2 \alpha_{21} y_{1} y_{2}^{2}+\alpha_{12} y_{2}^{3} \\ 3 \beta_{30} y_{1}^{2} y_{2}+2 \beta_{21} y_{1} y_{2}^{2}+\beta y_{2}^{3}\end{array}\right]+\left[\begin{array}{c}-y_{1}^{3}+y_{1} y_{2}^{2}-y_{2}^{3} \\ 3 y_{1}^{3}+y_{1}^{2} y_{2}\end{array}\right]$ $=$

$\left[\begin{array}{c}\left(\beta_{30}-1\right) y_{1}^{3}+\left(\beta_{21}-3 \alpha_{30}\right) y_{1}^{2} y_{2}+\left(\beta_{12}-2 \alpha_{21}+1\right) y_{1} y_{2}^{2}+\left(\beta_{03}-\alpha_{12}-1\right) y_{2}^{3} \\ 3 y_{1}^{3}+\left(-3 \beta_{30}+1\right) y_{1}^{2} y_{2}+\left(-2 \beta_{21}\right) y_{1} y_{2}^{2}+\left(-\beta_{12}\right) y_{2}^{3}\end{array}\right]$

If we choose that

$\beta_{30}=1, \beta_{21}=\beta_{12}=\alpha_{30}=0, \alpha_{21}=\frac{1}{2}$,

$\beta_{03}=\alpha_{12}+1$, and $\alpha_{03}$ is arbitrary, then

$\boldsymbol{g}_{3}(\boldsymbol{y})=\left[\begin{array}{c}0 \\ 3 y_{1}^{3}-2 y_{1}^{2} y_{2}\end{array}\right]$.

Based on relations (11) and (16), system (2) around the point $O(0,0)$ can be written in the form

$\left\{\begin{array}{c}\dot{x}=y+0\left(|x|^{4}\right) \\ \dot{y}=x^{2}+3 x^{3}-2 x^{2} y+0\left(|x|^{4}\right)\end{array}\right.$

as $|\boldsymbol{x}| \rightarrow 0$.

Based on all of the above, the following theorem holds

Theorem 2. For system (2) the equilibrium point $O(0,0)$ is a cusp.

Proof: The system (17) can be written in the form

$\left\{\begin{array}{c}\dot{x}=y+0\left(|x|^{4}\right) \\ \dot{y}=x^{2}(1+3 x)-2 x^{2} y+0\left(|x|^{4}\right),\end{array}\right.$

whence we see that it is

$\alpha_{k}=1, h(x)=3 x, k=2,(m=1), \beta_{n}=-2$,

$n=2, n \geq m$.

Thus all the conditions of Theorem 1 under a) are fulfilled, and on this basis we conclude that the point $O(0,0)$ is a cusp.

Using the approximate values of the equilibrium points $C(1.67,1.22), D(-1.50,1.53)$,

$E(-0.31,-0.12)$ the following theorem provides an analysis of their local stability.

Theorem 3. It holds

a) The equilibrium point $C(1.67,1.22)$ is a sink.

b) The equilibrium point $D(-1.50,1.53)$ is a spiral sink.

c) The equilibrium $E(-0.31,-0.12)$ is a saddle point.

Proof: The Jacobian matrices of these points are given in sequence by

$$
\begin{gathered}
D_{f}(C)=\left(\begin{array}{cc}
-3.53 & 0.61 \\
5.19 & -6.12
\end{array}\right), \\
D_{f}(D)=\left(\begin{array}{cc}
-7.40 & -10.61 \\
5.28 & 0.75
\end{array}\right), \\
D_{f}(E)=\left(\begin{array}{ll}
-0.89 & 1.03 \\
-0.16 & 0.52
\end{array}\right),
\end{gathered}
$$

from which we conclude that

$$
\begin{gathered}
\lambda_{1}(C) \cong-7.02, \lambda_{2}(C) \cong-2.62, \\
\lambda_{1,2}(D) \cong-3.32 \pm 6.27 i, \text { and } \\
\lambda_{1}(E) \cong-0.76, \lambda_{2}(E) \cong 0.39,
\end{gathered}
$$

whence the proof of the theorem follows, see [6], [7] and [8].

The Figures 4 and 5 show one trajectory weighing points $C(1.67,1.22)$ and $D(-1.50,1.53)$.

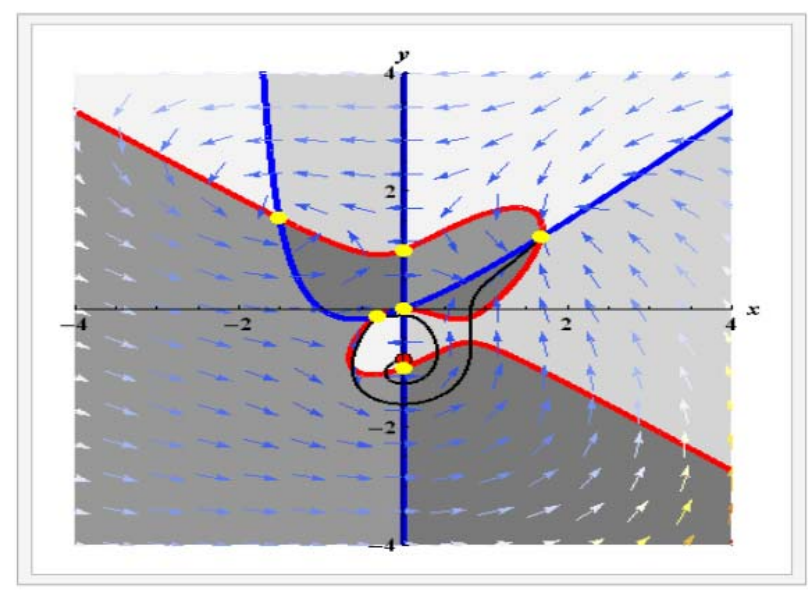

Figure 4. The point $\boldsymbol{C}(\mathbf{1} . \mathbf{6 7}, \mathbf{1 . 2 2})$ is real a sink

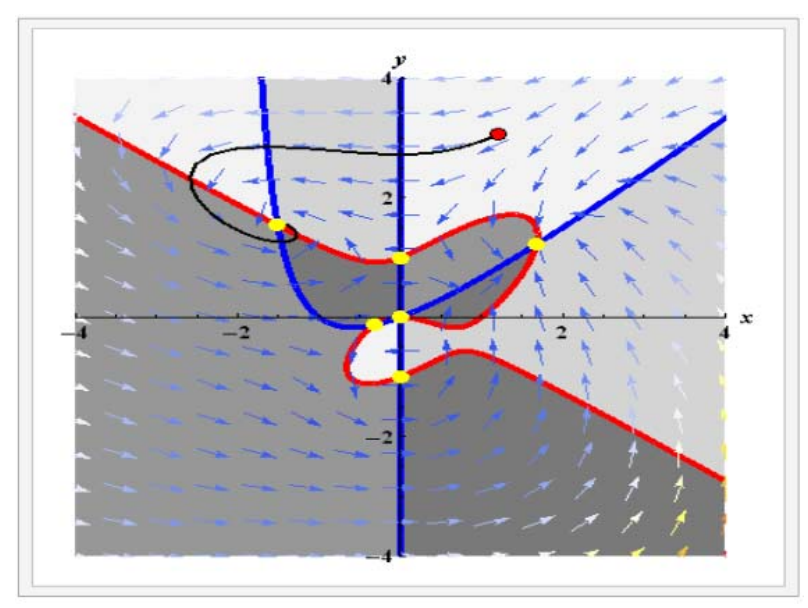

Figure 5. The point $\boldsymbol{D}(\mathbf{- 1 . 5 0 , 1 . 5 3 )}$ is a spiral sink

From the local dynamics, we can see that 2 of the 6 equilibrium points are stable and have adequate attraction basins. These basins are separated by a curve that is of central manifold and difficult to determine analytically. This curve is invariant with respect to the flow of system (2). 


\section{Conclusion}

In general, the procedure for testing the stability of non-hyperbolic equilibrium is not at all easy. Finding the Lyapun function is a heuristic approach that often fails. In recent times, Normal forms for nonlinear control systems with scalar output are increasingly being tested, see [9]. The Normal Form Theory and Center Manifold Theorem are used whenever possible to examine the stability of non-hyperbolic equilibriums.

The difficulty of performing this procedure in which the system is brought to normal form is in determining the near-identity transformation of coordinates. This procedure was first applied in the Ph.D thesis of Poincare, see [10]. For all other points, local stability was investigated in detail.

\section{References}

[1]. Venti, R. J. (1966). Linear normal forms of differential equations. Journal of Differential Equations, 2(2), 182-194.

[2]. Wu, C. (2007). Invariant closed surface and stability of non-hyperbolic equilibrium point for polynomial differential systems in R3. Journal of mathematical analysis and applications, 326(2), 1346-1355.

[3]. Xiao, M., \& Cao, J. (2009). Hopf bifurcation and nonhyperbolic equilibrium in a ratio-dependent predatorprey model with linear harvesting rate: Analysis and computation. Mathematical and Computer Modelling, 50(3-4), 360-379.

[4]. M.W. Hirsch, S. Smale, R.L. Devaney. (1974). Differential Equations Dynamical Systems, and An Introduction to Chaos (Elsevier, San Diego), 2nd Ed.

[5]. L. Perko (2002). Differential Equations and Dynamical Systems (third edition), Texts Appl. Math., vol. 7, Springer-Verlag.

[6]. Verhulst, F. (2006). Nonlinear differential equations and dynamical systems. Springer Science \& Business Media.

[7]. Strogatz, S. (2000). Nonlinear Dynamics and Chaos. Westview Press.

[8]. Wiggins, S. (2003). Chaos and Strange Attractors. Introduction to Applied Nonlinear Dynamical Systems and Chaos, 736-746.

[9]. Tall, I. A., \& Balde, M. (2005). Normal forms for nonlinear control systems with scalar output. Comptes Rendus Mathematique, 341(9), 573-578.

[10]. Poincaré, H. (1879). Sur les propriétés des fonctions définies par les équations aux différences partielles (No. 4). Gauthier-Villars. 